\title{
Experten vernetzen und diagnostisches Bildmaterial bündeln
}

Bilder versenden und befreundete Kollegen in besonderen Fällen, in denen man sich nicht ganz sicher ist, um ihre fachliche Meinung bitten - das ist lange schon Usus in der radiologischen $\mathrm{Ge}$ meinschaft. Allerdings findet der Austausch meist eher spontan und im eigenen Netzwerk statt. Doch es gibt Fälle, in denen man nicht den entsprechenden Spezialisten kennt, den man um Rat bitten könnte. Zudem wird es mit zunehmender Bilderflut und Informationsmenge immer schwieriger, geeignete Datensätze in großer Zahl zu versenden, da die technischen Hilfsmittel hierfür unzureichend sind. Unterstützung bietet seit Anfang Juli das neue „Expertennetzwerk“ der Deutschen Röntgengesellschaft. Die Hintergründe erklärt der stellvertretende DRG-Präsident Prof. Dr. Dierk Vorwerk (Ingolstadt) im Interview.

Der ein oder andere Radiologe tauscht sich ja bereits bei komplizierten Fällen mit Kollegen aus. Welchen Mehrwert bietet hierfür das Expertennetzwerk den DRG-Mitgliedern?

Prof. Dr. Vorwerk Wir wollen die Abläufe solcher Anfragen verbessern und unseren Mitgliedern einen Service bieten, über den sie auf passende Experten zurückgreifen können, um nicht allein auf persönliche Kontakte angewiesen zu sein. Auch möchten wir die Möglichkeit bieten, größere Datenmengen einfach zur Verfügung zu stellen, damit die Kollegen eine fundierte Einschätzung liefern können.

Wie kam es dazu, dass das Expertennetzwerk gerade jetzt gestartet wurde?

Wir haben im vergangenen Jahr gemeinsam mit der MeVis Medical Solutions AG sehr erfolgreich eine DRG-eigene webbasierte Fallsammlung konzipiert. Auf der Basis dieser guten Erfahrung und in ähnlichen Strukturen ist es nun möglich, ein Expertennetzwerk digital umzusetzen. Das Netzwerk kommt als Folgeprojekt also genau zum richtigen Zeitpunkt, da wir die technischen Möglichkeiten diesbezüglich weiter ausschöpfen konnten und sozusagen im Schwung sind. Auf dem 98. RöKo in Leipzig konnten sich interessierte Kongressteilnehmer erstmalig davon überzeugen, wie gut die neue Software zum webbrowserbasierten Austausch radiologischer Daten funktioniert. Und nun hoffen wir natürlich auf eine rege Nutzung dieses neuen DRGAngebots.

\section{Zu welchen Themen kann man sich im Expertennetzwerk Ratschläge und Tipps holen?}

In der Einführungsphase bis Ende 2017 fokussieren wir auf Herz- und Gefäßdiagnostik und Muskuloskelettale Radiologie. Wir planen aber, das Angebot sukzessive auf zusätzliche Fachgebiete zu erweitern. Übrigens sind während dieser Phase alle Anfragen kostenfrei.

Wie genau funktioniert eine Anfrage? Können Radiologen auch einen bestimmten Experten um Antwort bitten?

Unsere Experten sind nach Fachgebieten in Gruppen aufgeteilt. Ratsuchende Radiologen loggen sich mit ihren DRG-Zugangsdaten ein und wählen zunächst ein Fachgebiet aus. In ein Freitextfeld werden dann die Frage und weitere Informationen zum Patienten eingetragen. Außerdem können alle nötigen Unterlagen wie DICOM-Daten, JPG- oder PNG-Dateien hochgeladen werden. Der Fragesteller kann auch angeben, in welchem Zeitraum er eine Antwort benötigt - in dringenden Fällen ist das sogar innerhalb von 24 Stunden denkbar. Die Frage wird dann dem gesamten Fachgebiet zugänglich gemacht - oder einem einzelnen, vom Fragesteller bestimmten Experten.

Zukünftig wird die Erfassung und Auswertung von Bilddaten ausschließlich digital und mithilfe lernender Maschinen erfolgen. Denken Sie, dass der Radiologe als verantwortlicher Arzt künftig vom Computer ersetzt wird?

Auf keinen Fall! Aber das Arbeitsfeld wird sich, wie schon im Verlauf der letzten 50 Jahre, verändern. Der Radiologe von morgen wird rechnergestützte Diagnosesysteme in Anspruch nehmen, seine Diagnose dadurch verbessern, aber die kritische, erfahrungsbasierte Würdigung der Befunde

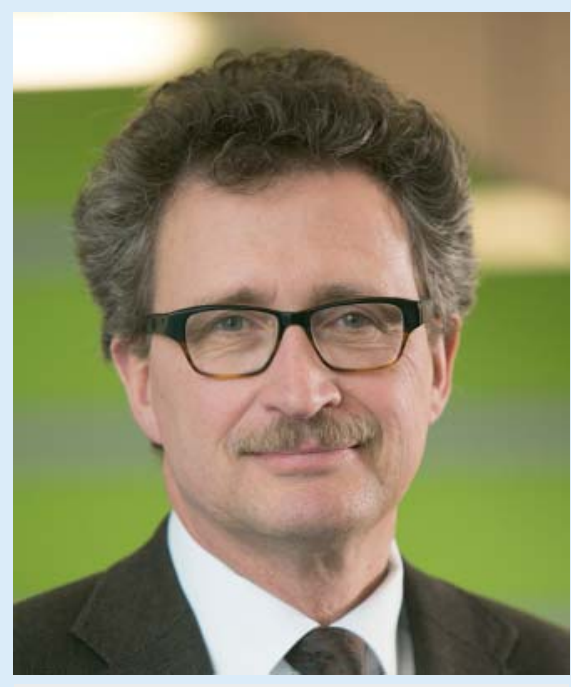

Prof. Dr. Dierk Vorwerk

wird unverändert beim Fachradiologen bleiben. Die Radiologie der Zukunft wird sicherlich auch klinischer sein, da sie vermehrt diagnostisch und interventionelltherapeutisch im klinischen Geschehen eines jeden größeren Krankenhauses eingreifen wird.

Wir müssen also keine Angst um den Radiologen, wie wir ihn kennen, haben?

Es spricht nichts dafür, Angst vor der Zukunft zu haben, denn Veränderungen finden ständig statt. Als vor 130 Jahren die Kutschen durch Automobile verdrängt wurden, hat das zwar die Pferde betroffen, nicht aber die Kutscher, war es doch immer noch notwendig jemanden zu finden, der das Gefährt - egal wie betrieben - steuern konnte. So wird es auch für die Radiologie weitergehen. Für Zukunftsängste besteht kein Anlass.
SO FUNKTIONIERT DAS
EXPERTENNETZWERK
1. Logln mit den DRG-Mitgliederdaten
2. Fachgebiet auswählen, ggf. gezielt Experten auswählen
3. Frage formulieren, Daten hochla- den, Antwortzeitraum bestimmen
4. Antwort online einsehen 